\title{
Diabetic Foot Care Knowledge and Behaviors of Individuals with Diabetes Mellitus in Indonesia
}

\author{
Sulistyo AHS ${ }^{1}$,Sae-Sia W' ${ }^{2}$ \& Maneewat $\mathrm{K}^{3}$ \\ ${ }^{1}$ Master of Nursing Student, Faculty of Nursing, Prince of Songkla University, Hat Yai, Songkhla, \\ Thailand \\ ${ }^{2,3}$ Assistant Professor of Nursing, Faculty of Nursing, Prince of Songkla University, Hat Yai, Songkhla, \\ Thailand
}

Corresponding author email: angger.anugerah@gmail.com

\begin{abstract}
The most common diabetes mellitus (DM) complication is diabetic foot ulcer (DFU), which is costly and has devastating adverse effects. It was found that 6.8 $\%$ of DM patients suffered from DFU. According to Indonesian Hospital Association, DFU was reported to cause an amputation rate of $15-30 \%$. The aims of this study were to assess the levels of diabetic foot care knowledge and behaviors and its relationship among diabetic patients attending Public Health Center at Bojonegoro, East Java, Indonesia. Eighty-one participants with DM were selected from two public health centers based on inclusion criteria. Modified Diabetic Foot Care Knowledge and Modified Diabetic Foot Care Behaviors Questionnaires were used in this study. The diabetic foot care knowledge and behaviors were categorized into 3 levels as poor $(<60 \%)$, moderate $(60-80 \%)$, and good $(>80 \%)$. Data were analyzed by descriptive statistics. Pearson correlation coefficient was employed to analyze the correlation between diabetic foot care knowledge and behaviors among participants. Based on the result, the diabetic foot care knowledge score was at poor level $(M=57.8, S D=14.6)$ of which $39.5 \%$ of them had poor knowledge, $58 \%$ had moderate and only $2.5 \%$ had good knowledge. Diabetic foot care behaviors was at poor level $(M=46.7, S D=10.9 \%)$ of which $86.4 \%$ of them had poor behaviors and the remaining of $12.3 \%$ had moderate behaviors. The correlations between diabetic foot care knowledge and behaviors were not significantly correlated $(p=.09)$. The diabetic foot care knowledge and behaviors are still become the biggest concern among diabetic's patients in Indonesia. This study showed that DM patients with good knowledge of DM foot care might not always transform their knowledge into practices. Further research is needed to enhance diabetic foot care
\end{abstract}

DOI: $10.5176 / 2345-7198 \_5.1 .4$

ISSN 2345-7198

(CThe Author(s) 2018. This article is published with open access by the GSTF knowledge and behaviors among DM patients in Indonesia.

Keywords: Diabetes Mellitus, Diabetic Foot Ulcer, Diabetic Foot Care Knowledge, Diabetic Foot Care Behaviors

\section{INTRODUCTION}

Diabetes mellitus (DM) is an increasing global health issue. Around 387 million people worldwide were diagnosed with DM [1]. In South East Asia, it affected 78 million people and has been predicted to increase to 140 million cases by 2040 [1]. DM is in the top 10 diseases that cause death in Indonesia and a recent study found 9 million Indonesian patients suffering from DM [2].

DM leads to some serious complications including microvascular complications (impaired vision, renal failure, loss of sensation, and ulceration) and macrovascular complications (myocardial infarction, stroke, and ischemia) [3]. The most common DM complication is diabetic foot ulcer (DFU), which is costly and has devastating adverse effects [4]. It is estimated that more than $25 \%$ of DM patients suffered from DFU during their lifetime [5]. Around $6.8 \%$ of DM patients suffered from DFU [6]. Annually, 1 million limb amputations worldwide were due to DFU and in the United States, DFU led to 80.000 amputations per year [7]. Although there are no reported studies regarding the number of amputations due to DM in Indonesia but based on data from the Indonesian Hospital Association, DFU was reported to 
cause a mortality rate of $17-23 \%$ and an amputation rate of $15-30 \%$ [8].

Although a large amount of articles regarding foot care knowledge and behaviors published, there is limited literature published on foot care knowledge and behaviors particularly in Indonesia. Therefore, the study aimed to examine the current prevalence and the relationship between foot care knowledge and behaviors on diabetics' patients who attend monthly general checkup at public health centers at Bojonegoro, East Java, Indonesia. The result of this study would inform the current level of foot care knowledge and behaviors at that particular area.

\section{METHODS}

This study was descriptive correlational study, which included 81 participants of diabetic patients at Bojonegoro, East Java, Indonesia. Data were gathered from two biggest public health centers populations of diabetes patients named Bojonegoro Public Health Center and Ngumpakdalem Public Health Center. The inclusion criteria of (1) over 18 - 65 years old; (2) diagnosed with diabetes mellitus by the physician; (4) be able to do daily activity independently; (5) no existing visual problem; (6) no existing hearing problem; and (8) agreeing to participate in the study were applied in this study. Participants who agreed to join in this research were directly examined their knowledge and behaviors regarding foot care before scheduled general check-up be done.

Two questionnaires were used in this study including Modified Diabetic Foot Care Knowledge (MDFCK) and Modified Diabetic Foot Care Behaviors (MDFCB). The MDFCK and MDFCB were validated by three experts. The reliability test found that the MDFCK questionnaire yielded a KR-20 coefficient of .75 and the MDFCB yielded a Cronbach's alpha coefficient of .81 .

\section{A. Modified Diabetic Foot Care Knowledge Questionnaire (MDFCK).}

The questionnaire of MDFCK was used in this study to determine the level of diabetic foot care knowledge. The questions in this questionnaire were used to measure basic knowledge regarding foot care. This questionnaire consisted of 15 items including general diabetes managements (5 items), preventing foot injuries (2 items), checking foot condition (2 items), foot hygiene (3 items), appropriate footwear (2 items), and toenail care (1 items). Each item was measured by using true/false question in which the " 1 " point for the correct answer and " 0 " point for the wrong answer.

The formula to calculating the score was,

Standard score $=($ actual score/maximum score $) * 100$

The standard score of foot care knowledge described of poor $(<60)$, medium $(60-80)$ and good $(>80)$ [9].

\section{B. Modified Diabetic Foot Care Behaviors Questionnaire (MDFCB).}

The diabetic foot care behaviors was measured by MDFCB questionnaire. The questionnaire consisted of 34 items including general diabetes management (4 items), checking foot condition (4 items), foot hygiene (4 items), appropriate footwear (11 items), foot moisturize (2), toenail care (5), foot injury prevention (1 items), and foot injuries treatment (3 items). Each item is measured using a (0-3) Likert scale in which the positive statement " 0 " represents "never practice" and " 4 " represents "always practice", and on the other hand the negative statement applied the opposite scoring.

The formula to calculating the score was,

Standard score $=($ actual score/maximum score $) * 100$

The standard score of foot care behaviors described of poor $(<60)$, medium $(60-80)$ and good $(>80)$ [9].

\section{Statistical Analytics}

The IBM SPSS Statistics package version 19 was used to analyze all data in this study. Pearson correlation coefficient was employed to analyze the correlation between diabetic foot care knowledge and behaviors among participants. Mean and standard deviation were used to present the data and $p$-value $<.05$ was considered to determine the significant value.

\section{FINDINGS}

\section{A. Characteristics of Participants}

A total of 81 participants from two public health centers at Bojonegoro were included in this study. As 
shown in Table 1, the mean age of participants was 55.51 years $(S D=7.3)$. Majority of participants were female $(n=58,71.6 \%)$. All of the participants of this study is a Muslim $(n=81,100 \%)$. The majority of participants had studied to elementary school level ( $n=31,38.3 \%$ ). Based simple examination, most of all participants did not have sign of foot problem including, neuropathy signs $(n=50,61.7 \%)$, PAD signs $(n=66,81.5 \%)$, and foot deformity $(n=79$, $97.5 \%)$.

\begin{tabular}{l} 
TABLE 1. DEMOGRAPHIC CHARACTERISTIC OF PARTICIPANTS \\
\begin{tabular}{|l|c|}
\hline \multicolumn{1}{|c|}{ Participants' characteristics } & $\begin{array}{c}\text { Total } \\
\text { participants } \\
\text { (N= 81) }\end{array}$ \\
\hline Age, $M(S D)$ & $55.51(7.3)$ \\
Gender $n(\%)$ & $23(28.4)$ \\
Male & $58(71.6)$ \\
Female & \\
Religion & $81(100)$ \\
Islam & $31(38.3)$ \\
Education Status $n(\%)$ & $23(28.4)$ \\
Elementary School & $14(17.3)$ \\
Junior High School & $7(8.6)$ \\
Senior High School & $6(7.4)$ \\
Bachelor Degree & $22.9(3.1)$ \\
No Formal Education & $201(81.4)$ \\
BMI M(SD) & \\
Latest Blood Glucose M(SD) & $20(24.7)$ \\
DM Duration $n(\%)$ & $29(35.8)$ \\
$<1$ year & $32(39.5)$ \\
1-5 years & \\
$>5$ years & $50(61.7)$ \\
Neuropathy Sign $n(\%)$ & $31(38.3)$ \\
No & \\
Yes & $66(81.5)$ \\
PAD (peripheral arterial disease) Sign $n(\%)$ & $15(18.5)$ \\
No & $79(97.5)$ \\
Yes & $2(2.5)$ \\
Foot Deformity $n(\%)$ & \\
No & \\
Yes & \\
\hline Note: $M=$ Mean, $S D=$ Standard Deviation & \\
& \\
\hline
\end{tabular} \\
\hline
\end{tabular}

\section{B. Diabetic Foot Care Knowledge (DFCK)}

Table 2 showed the level of diabetic foot care knowledge among participants. The participants could obtain the score of foot care knowledge ranged from $0-15$. The majority of participants had a moderate level of knowledge (58\%). Participants with a poor level of knowledge were $39.5 \%$ and the rest had a good level $(2.5 \%)$.

\section{Diabetic Foot Care Behaviors (DFCB)}

The possible score that participants can obtain on diabetic foot care behaviors ranged 0-102. The mean of diabetic foot care behaviors was at poor level $(M=$ $46.7, S D=10.9$ ), of which $86.4 \%$ of them had poor behaviors and the remaining of $13.6 \%$ had moderate behaviors.
TABLE 2. THE FREQUENCIES AND PERCENTAGE OF PARTICIPANTS CATEGORIZED BY LEVELS OF DFCK AND DFCB

\begin{tabular}{|c|l|l|l|}
\hline Items & \multicolumn{1}{|c|}{ Poor level } & \multicolumn{1}{|c|}{ Medium level } & Good level \\
\hline DFCK $n(\%)$ & $32(39.5)$ & $47(58)$ & $2(2.5)$ \\
DFCB $n(\%)$ & $70(86.4)$ & $11(13.6)$ & $0(0)$ \\
\hline
\end{tabular}

\section{Relationship Between Diabetic Foot Care Knowledge and Behaviors}

Based on correlation analysis, there was no significant correlation between diabetic foot care knowledge and diabetic foot care behaviors $(p=.09)$.

\section{E. Discussion}

Diabetic foot care knowledge was related to the development of diabetic foot ulcer (DFU). Adequate knowledge regarding diabetic foot care proved reducing the development of diabetic foot complications, particularly foot ulcer [10]. In this current study, the majority of participant had limited information regarding foot ulcer prevention. It was be evidenced with, the majority of participants had poor to moderate level of diabetic foot care knowledge. Although there are several monthly programs aimed diabetics' patients in the area of the study including vital sign examination, blood glucose checkup, medication, health education (medication used, exercise and diabetic diet), and foot care consultation (if participants complain of neuropathic symptoms or when the physician notices any foot deformities) but limited face to face interaction with health practicioner seems to be an issue. At the study setting, it seemed difficult to arrange the proper time for face to face educational session because currently, only one or two health practitioners take in charge role while many patients are queuing for medication. One systematic review suggested that more interaction between participants and health care provider was pivotal in a foot care prevention program [11].

Based on the result of this study, the majority of participants' diabetic foot care knowledge were at moderate level. This result was congruent with the latest study from Central Java of Indonesia that the majority $(56 \%)$ of the diabetic foot care knowledge was on the moderate level [12]. In comparison with developed country, the study at Saudi Arabia showed that $98.7 \%$ of 269 participants in the study had good knowledge regarding diabetic foot care [13]. This dissimilarity of diabetic foot care knowledge result could be because of the disparity of human resources and health care facilities available in those countries. 
There were several participants' characteristics that could influence the result of diabetic foot care knowledge. Those characteristics are age and gender [14], and level of education [15]. Mean age of participants were at middle age adult (55.51 years old) that associated with degeneration and decreased or deranged physiological function. Since the level of diabetic foot care knowledge is the ability of participants to understand and remember the information regarding DFU prevention, the aging process believed influencing the cognitive function to understand and remember the information and instruction. Based on adult learning theory, aging process impacted physical changes, such as reduced hearing and vision ability and age-related factors problem, such as impaired blood circulation, decreased neurotransmitter, and chronic illness [16]. In addition, the level of education potentially influenced the result of diabetic foot care knowledge of participants. More than half of participants had studied in elementary school and junior high school. The study of Chellan et al. (2012) proved that participants who graduated from high school had lower foot care knowledge than those graduated from the bachelor degree. Moreover, the participants who have a higher level of education showed the low incident of DFU [15].

Knowledge, as well as good behaviors of the participants, are needed in order to prevent foot ulcer. Based on the result of this current study, $86 \%$ of participants had the poor level of diabetic foot care behaviors. It means, 70 participants did not perform diabetic foot care behaviors properly. This result could be influenced by poor habits regarding foot care, such as improper diabetic diet, barefoot walking, and inappropriate toenail care that were common in the study settings. The result of this study was consistent with the study in China that majority of participants had the poor level of diabetic foot care behaviors [9].

The Pearson correlation coefficient showed, no significant relationship between diabetic foot care knowledge and diabetic foot care behaviors. This result revealed that participants with a good level of diabetic foot care knowledge not always showed a good level of diabetic foot care behaviors or vice versa. That evidence showed that patients with adequate knowledge regarding DFU prevention did not always translate their knowledge into desired foot care behaviors. The result of this study was congruent with several studies [17] [18] that showed no correlation between diabetic foot care knowledge and diabetic foot care behaviors.
However, strategies to enhance diabetic foot care knowledge and diabetic foot care behaviors still remained important. It needs several improvements of educational strategies in order to maintain participants' knowledge and behaviors regarding foot care. One systematic review reported that the followup strategies, such as a telephone call were effective and feasible to assess knowledge, discuss the patients' concerns, and encourage lifestyle and behavioral changes [19].

\section{CONCLUSION}

Based on our study result, the diabetic foot care knowledge and behaviors among participants in this study were not adequate. The gaps of inadequate diabetic foot care knowledge and behaviors of participants indicated the need of interactive educational intervention regarding foot care. In order to minimize the complications of diabetic foot, health practitioners who directly contact with DM patients should be organized appropriate health education regarding foot care.

\section{ACKNOWLEDGEMENT}

We would like to thank Thailand's educations hub for the southern region of ASEAN countries (TEHAC) Scholarship that gives support funding for study and research.

\section{REFERENCE}

[1] International Diabetes Federation. (2014). Indonesia. Retrieved from https://www.idf.org/membership/wp/indonesia

[2] Centers for Disease Control and Prevention. (2015, May 15). 2014 National diabetes statistics report. Retrieved November 10, 2015. Retrieved from http://www.cdc.gov/diabetes/data/statistics/2014statisticsrepo rt.html

[3] Walker. B. R.. Colledge. N. R.. Ralston. S. H.. \& Penman. I. (2013). Davidson's principles and practice of medicine. Philadelphia, PA: Elsevier Health Sciences.

[4] Yazdanpanah, L., Nasiri, M., \& Adarvishi, S. (2015). Literature review on the management of diabetic foot ulcer. World .Journal of Diabetes, 6(1), 37. doi: 10.4239/wjd.v6.i1.37

[5] Armstrong, D. G., Wrobel, J., \& Robbins, J. M. (2008). Guest Editorial: are diabetes-related wounds and amnutations worse than cancer?. International Wound Journal, (4), 286-7. doi: 10.1111/j.1742-481X.2007.00392.X

[6] Cronenwett, J. L., \& Johnston, K. W. (2014). Rutherford's vascular surgery $8^{\text {th }}$ ed. Philadelphia, PA: Elsevier Health Sciences

[7] Aumiller. W. D.. \& Dollahite. H. A. (2015). Pathogenesis and management of diabetic foot ulcers. Iournal of the American Academy of Physician Assistants, 28(5), 28-34. 10.1097/01.JAA.0000464276.44117.b1 
[8] Indonesian Hospital Association [PERSI]. (2011). Neuropati diabetik menyerang lebih dari $50 \%$ penderita diabetes. Retrieved from http://www.pdpersi.co.id/content/news.php?catid=23

[9] Li, R., Yuan, L., Guo, X. H., Lou, Q. Q., Zhao, F., Shen, L., ... \& Sun, Z. L. (2014). The current status of foot self-care knowledge, behaviours, and analysis of influencing factors in patients with type 2 diabetes mellitus in China. International Journal of Nursing Sciences, 1(3), 266-271. doi: 10.1016/j.ijnss.2014.05.023

[10] Muhammad-Lutfi, A. R., Zaraihah, M. R., \& Anuar-Ramdhan, I. M. (2014). Knowledge and practice of diabetic foot care in an in-patient setting at a tertiary medical center. Malaysian Orthopaedic Journal, 8(3), 22. doi: 10.5704/MOJ.1411.005

[11] Bazian Ltd. (2005). Education to prevent foot ulcers in diabetes. Evidence-Based Healthcare and Public Health.2005;9:351-358. Retrieved from http://pmmp.cnki.net/Resources/CDDPdf/evd\%5Cbase\%5CE vidence-

based $\% 20$ Healthcare $\% 20$ and $\% 20$ Public $\% 20$ Health $\% 5 \mathrm{C} \% \mathrm{E} 7$ $\%$ B3\%BB\%E7\%BB\%9F\%E8\%AF\%84\%E4\%BB\%B7\%5Ce bhph20050905351.pdf.

[12] Nurhayati, I. (2017). Peningkatan Motivasi Foot Care Behavior Melalui Teknik Edukasi Pada Penderita Diabetes Melitus Di Keluarga. IJMS-Indonesian Journal on Medical Science, 4(1).

[13] Alrajeh, A. (2016). Assessment of knowledge and practice of diabetic foot care among diabetic patients and primary care physicians at a teaching hospital, Saudi Arabia. J Diabetes Metab, 7:11. doi : 10.4172/2155-6156.C1.061

[14] Desalu, O. O., Salawu, F. K., Jimoh, A. K., Adekoya, A. O., Busari, O. A., \& Olokoba, A. B. (2011). Diabetic foot care: self-reported knowledge and practice among patients attending three tertiary hospital in Nigeria. Ghana Medical Journal, 45(2), 60-65. Retrieved from https://www.ncbi.nlm.nih.gov/pmc/articles/PMC3158533/

[15] Chellan, G., Srikumar, S., Varma, A. K., Mangalanandan, T. S., Sundaram, K. R., Jayakumar, R. V., ... \& Kumar, H. (2012). Foot care practice-the key to prevent diabetic foot ulcers in India. The Foot, 22(4), 298-302. doi: 10.1016/j.foot.2012.08.007

[16] Merriam, S. B (Ed). (2001). The new update on adult learning theory. San Francisco: Jossey-Bass.

[17] Monami, M., Zannoni, S., Gaias, M., Nreu, B., Marchionni, N., \& Mannucci, E. (2015). Effects of a short educational program for the prevention of foot ulcers in high-risk patients: a randomized controlled trial. International Journal of Endocrinology, 2015. doi: 10.1155/2015/615680

[18] Pérez-Borges, G., Ledesma-Machado, J., Espinosa-Ramos, O., Mesa-Correa, C. D., Tort-Cursellas, L., \& HernándezHernández, L. (2015). The effect of an educational program using a group-based workshop to improve long-term care adherence in diabetic patients at risk of foot lesions. Journal of Nursing Education and Practice, 5(4), 122. doi: $10.5430 /$ jnep.v5n4p122

[19] Furuya, R. K., Mata, L. R., Veras, V. S., Appoloni, A. H., Dantas, R. A., Silveira, R. C., \& Rossi, L. A. (2013). Original research: telephone follow-up for patients after myocardial revascularization: a systematic review. AJN The American Journal of Nursing, 113(5), 28-31. doi: 10.1097/01.NAJ.0000429756.00008.ca 